\title{
Interplay of Magnetization Dynamics with a Microwave Waveguide at Cryogenic Temperatures
}

\author{
I.A. Golovchanskiy,,${ }^{1,2,}{ }^{*}$ N.N. Abramov, ${ }^{2}$ M. Pfirrmann, ${ }^{3}$ T. Piskor, ${ }^{3}$ J.N. Voss, ${ }^{3}$ D.S. Baranov,,${ }^{1,4,5}$ \\ R.A. Hovhannisyan, ${ }^{1}$ V.S. Stolyarov,,${ }^{1,4,6}$ C. Dubs, ${ }^{7}$ A.A. Golubov, ${ }^{1,8}$ V.V. Ryazanov, ${ }^{2,4,6}$ \\ A.V. Ustinov, ${ }^{2,3}$ and M. Weides ${ }^{3,9, \dagger}$ \\ ${ }^{1}$ Moscow Institute of Physics and Technology, National Research University, 9 Institutskiy Pereulok, Dolgoprudny, \\ Moscow Region, 141700, Russia \\ ${ }^{2}$ National University of Science and Technology MISIS, 4 Leninsky Prospekt, Moscow, 119049, Russia \\ ${ }^{3}$ Physikalisches Institut, Karlsruhe Institute of Technology, 76131 Karlsruhe, Germany \\ ${ }^{4}$ Institute of Solid State Physics of the Russian Academy of Sciences, Chernogolovka, Moscow Region, \\ 142432, Russia \\ ${ }^{5}$ Laboratoire de Physique et d'Etude des Materiaux, UMR8213, École Supérieure de Physique et de Chimie \\ Industrielles de la Ville de Paris, Paris Sciences et Lettres Research University, Institut des NanoSciences de \\ Paris-Sorbonne Universite, 10 rue Vauquelin, 75005 Paris, France \\ ${ }^{6}$ Solid State Physics Department, Kazan Federal University, Kazan, 420008, Russia \\ ${ }^{7}$ INNOVENT e.V. Technologieentwicklung Jena, Prüssingstraße 27B, 07745 Jena, Germany \\ ${ }^{8}$ Faculty of Science and Technology and MESA + Institute for Nanotechnology, University of Twente, \\ 7500 AE Enschede, Netherlands \\ ${ }^{9}$ School of Engineering, University of Glasgow, Rankine Building, Oakfield Avenue, Glasgow, G12 8LT, \\ United Kingdom
}

(Received 3 January 2019; revised manuscript received 7 February 2019; published 23 April 2019)

In this work, magnetization dynamics is studied at low temperatures in a hybrid system that consists of a thin epitaxial magnetic film coupled with a superconducting planar microwave waveguide. The resonance spectrum was observed over a wide magnetic field range, including low fields below the saturation magnetization and both polarities. Analysis of the spectrum via a fitting routine we develop allows the derivation of all magnetic parameters of the film at cryogenic temperatures, the detection of waveguideinduced uniaxial magnetic anisotropies of the first and the second order, and the uncovering of a minor misalignment of the magnetic field. A substantial influence of the superconducting critical state on the resonance spectrum is observed and discussed.

DOI: 10.1103/PhysRevApplied.11.044076

\section{INTRODUCTION}

The field of magnonics studies the application of magnetization oscillations and waves in ferromagnetic structures [1-6]. The following benefits make magnonics promising for application in processing of microwave signals: tunability of the magnon dispersion with applied magnetic field and the geometry of the medium, low dissipation and power consumption, high operational frequencies, convenient micron and submicron scales of the spin wavelength at microwave frequencies, and the absence of parasitic coupling of spin waves with nonmagnetic environments. Conventionally, magnonics is a room-temperature research discipline.

*golov4anskiy@gmail.com

†Martin.Weides@glasgow.ac.uk
Currently a subdiscipline is emerging that deals with magnetization dynamics at cryogenic temperatures and can be referred to as "cryogenic magnonics." Quantum magnonics is of high current interest [7-11]. Microwave experiments in quantum magnonics are typically performed at millikelvin temperatures, often using setups equipped with superconducting quantum circuits. On the other hand, various hybrid devices based on superconducting resonators [7,12] and Josephson junctions [13-15] are being developed. Also, it was shown that hybridization of a magnon medium with superconducting structures results in substantial modification of dispersion properties [16-19], as well as in the formation of magnonic band structures [20]. Finally, metamaterial properties have been reported for superconductor/ferromagnet superlattices [21]. More generally and beyond superconductorinduced phenomena, the magnetic properties at low temperatures are probed in the absence of or with only minor 
thermal excitations. Typical thermal effects for standard magnonics, such as reduced saturation magnetization or thermally activated domain-wall motion, are lessened for cryomagnonics, leading to new phenomena in ferromagnetic resonance (FMR).

In this regard, investigation of magnetic properties of ferromagnetic films at low temperature as well as of their interaction with superconducting circuits is imperative. This report addresses both problems. We focus on the ferromagnetic resonance in a thin yttrium iron garnet (YIG) film coupled to a superconducting $\mathrm{Nb}$ planar waveguide in an out-of-plane magnetic field. We obtain the FMR spectrum at low temperature in a wide field range. The spectrum shows linear magnetic resonance versus field dependence for high fields and a range of nonlinear dependence of the FMR frequency at low magnetic fields, where the Kittel formula is inapplicable. Developing a fitting routine, we derive all magnetic parameters of the YIG film. Our analysis shows that the waveguide itself induces substantial uniaxial magnetic anisotropy. Next, we study the FMR spectrum at temperatures below the superconducting critical temperature of the waveguide and observe an influence of the superconducting critical state of $\mathrm{Nb}$ on the resonance spectrum.

While YIG is probably the most-popular magnetic material for magnonic applications, owing to its low damping, the damping in YIG and its temperature dependence are not addressed in this paper and can be found elsewhere [22-24]. In this paper, YIG is selected as a model magnetic single-crystal thin film with distinct magnetocrystalline anisotropy and sufficiently low saturation magnetization, which is convenient for out-of-plane measurements.

\section{EXPERIMENTAL DETAILS}

The FMR absorption measurements are performed by the so-called vector-network-analyzer FMR (VNA-FMR) approach [25-27]. A schematic illustration of the system investigated is shown in Fig. 1. The single-crystal epitaxial YIG film of thickness $d=51 \mathrm{~nm}$ is deposited on a single-crystal [111]-oriented gadolinium gallium garnet (GGG) substrate by means of liquid-phase epitaxy (LPE). Details of LPE as well as the room-temperature characteristics of LPE-grown ultrathin YIG films can be found elsewhere $[23,28]$. Measurement of the FMR response in YIG is enabled by fabrication of a coplanar waveguide (CPW) directly on top of YIG film. The CPW is patterned out of 150-nm-thick magnetron-sputtered $\mathrm{Nb}$ thin film with superconducting critical temperature $T_{c} \simeq 8.5 \mathrm{~K}$ by photolithography and plasma-chemical etching. Deposition of $\mathrm{Nb}$ at room temperature is obstructed by poor adhesion of the metal film to the YIG surface, and therefore is performed at $300^{\circ} \mathrm{C}$. The $50-\Omega$ impedance of the superconducting $\mathrm{CPW}$ is provided by its gap-center-gap dimensions of 27, 40, and $27 \mu \mathrm{m}$. Direct placement of the

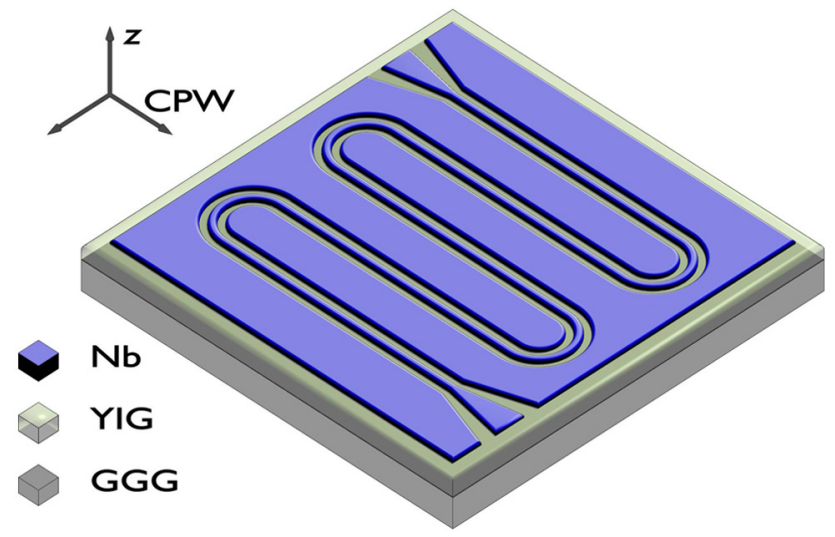

FIG. 1. The system investigated. YIG epitaxial film is grown on a [111]-oriented single-crystal GGG substrate by means of LPE. A Nb CPW is fabricated directly on top of the YIG film. The main direction of the $\mathrm{CPW}$ is indicated by the axis. A magnetic field $H$ is applied out of the plane, along the [111] orientation of YIG/GGG (i.e., along the $z$ direction).

$\mathrm{CPW}$ on the probed magnetic film and its elongation via meandering enhance sensitivity to weak FMR absorptions [29]. The experimental chip is installed in a copper sample holder and wire bonded to a printed circuit board with rf connectors. A thermometer and a heater are attached directly to the holder for precise temperature control. The holder is placed in a superconducting solenoid inside a closed-cycle cryostat (Oxford Instruments, Triton, base temperature $1.2 \mathrm{~K}$ ). The response of the system is studied analyzing the transmitted microwave signal $S_{21}$ with a Rohde \& Schwarz ZVB20 vector network analyzer.

\section{RESULTS AND DISCUSSION}

Figures 2(a) and 2(b) show transmission spectra of the sample studied at $T=10 \mathrm{~K}>T_{c}$ of $\mathrm{Nb}$ and at $2 \mathrm{~K}<$ $T_{c}$. The spectra have been normalized with $S_{21}(f)$ at $\mu_{0} H=0.5$ T. Figures 2(c) and 2(d) show a set of normalized absorption curves $S_{21}(f)$ of the sample at the same temperatures and several magnetic fields. Field-dependent spectral lines in Figs. 2(a) and 2(b) with the minimum transmission correspond to FMR curves $f_{r}\left(\mu_{0} H\right)$. Both spectra show linear FMR response at $\left|\mu_{0} H\right|>0.2 \mathrm{~T}$, which is typical for the Kittel FMR mode of a thin film in an out-of-plane magnetic field. The resonance frequency with an out-of-plane field is $f_{r} \propto\left(\mu_{0} H-4 \pi M_{\text {eff }}\right)$, which indicates the value of the effective saturation magnetization $4 \pi M_{\text {eff }} \sim 2000$ Oe at $f_{r} \rightarrow 0$. When $\left|\mu_{0} H\right|$ is decreased, the linear resonance line is terminated with a kink at $\left|\mu_{0} H\right| \sim 4 \pi M_{\text {eff }}$ and transforms into two FMR branches with nonlinear dependence of resonance frequency versus field for $\left|\mu_{0} H\right|<4 \pi M_{\text {eff. }}$ We refer to the higherfrequency FMR branch, with stronger absorption, as the "C line" and to the lower-frequency FMR branch, with 

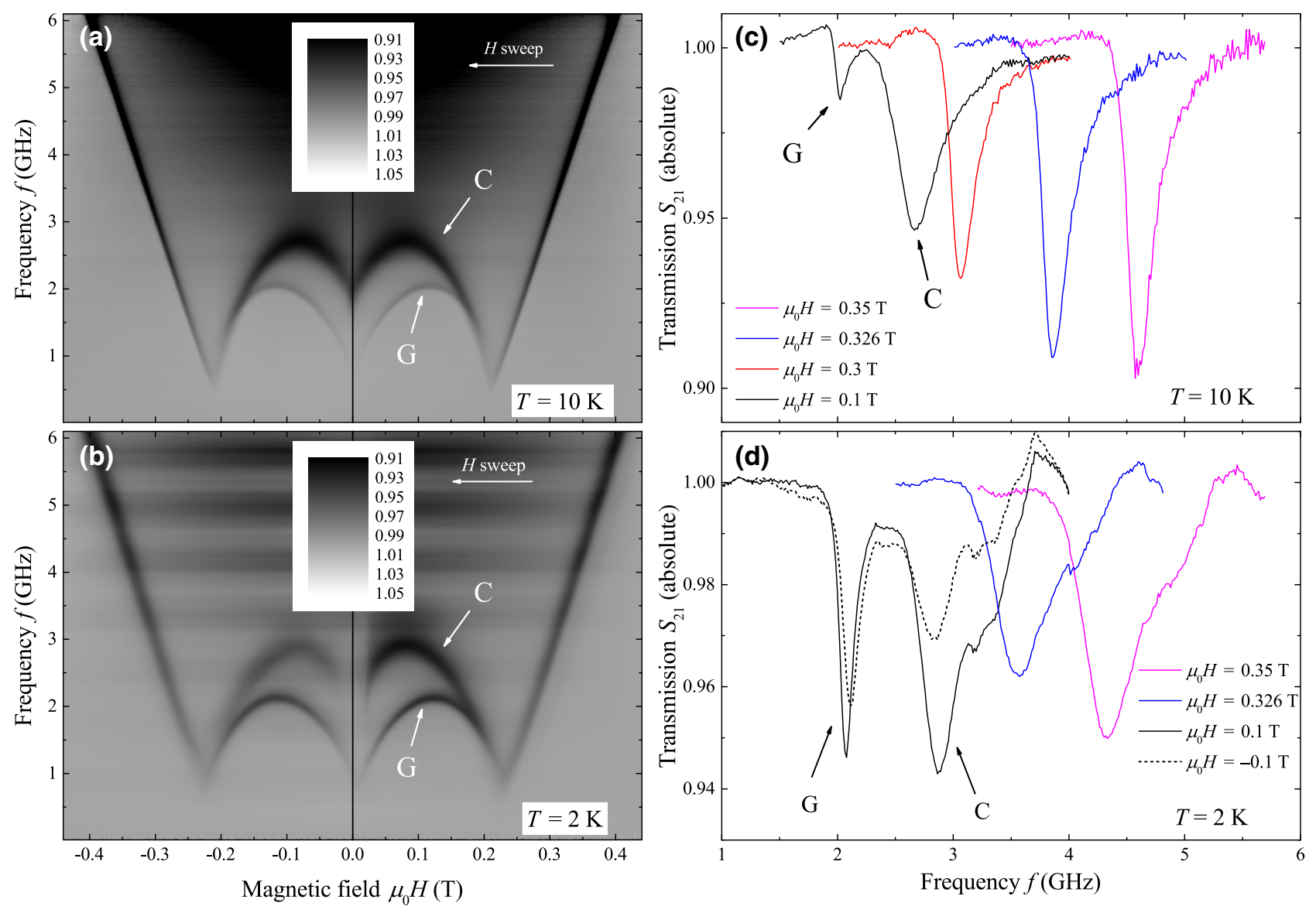

FIG. 2. (a),(b) Gray-scale-coded transmission spectra $\left|S_{21}\left(\mu_{0} H, f\right) / S_{21}\left(\mu_{0} H=0.5 \mathrm{~T}, f\right)\right|$ measured at $T=10 \mathrm{~K}$ above $T_{c}$ (a) and $T=2 \mathrm{~K}$ below $T_{c}$ (b). (c),(d) Corresponding frequency dependencies of the normalized transmission $\left|S_{21}(f)\right|$ at several magnetic fields at $T=10 \mathrm{~K}$ (c) and $T=2 \mathrm{~K}$ (d). For the curves in (c),(d) the background is subtracted. At $T<T_{c}$ the spectrum shows hysteresis of absorption. The magnetic field is swept negatively from 0.5 to $-0.5 \mathrm{~T}$ (indicated by arrows), and therefore the part of the spectrum in (b) at positive fields provides the "down-field-sweep" data, while the part of the spectrum at negative fields provides the "up-field-sweep" data. $\mathrm{C}$ and $\mathrm{G}$ indicate higher- and lower-frequency spectral lines, respectively.

weaker absorption, as the "G line." In general, observation of FMR in thin films with out-of-plane geometry at $\left|\mu_{0} H\right|<4 \pi M_{\text {eff }}$ might be challenging due to formation of nonuniform magnetization configurations. With an out-ofplane field $\left|\mu_{0} H\right|<4 \pi M_{\text {eff, ferromagnetic films are not }}$ magnetized to saturation, and the Kittel formula is not applicable. Splitting of the FMR response into several spectral lines at $\left|\mu_{0} H\right|<4 \pi M_{\text {eff }}$ can be caused by various factors, including standing-spin-wave resonances $[18,30$ 32], FMR response of magnetic domain structure [33-35], and magnetic phase separation.

After transition of the $\mathrm{Nb} \mathrm{CPW}$ into the superconducting state, the transmission spectrum changes [compare Figs. 2(a) and 2(b) and Figs. 2(c) and 2(d)]. While the spectrum at $T<T_{c}$ consists of the same resonance lines as the spectrum at $T>T_{c}$, superconductivity manifests itself in hysteresis of FMR peak absorption at $\left|\mu_{0} H\right|<0.2 \mathrm{~T}$, which is best visible for the C line [compare $S_{21}(f)$ at $\mu_{0} H=0.1 \mathrm{~T}$ and $\mu_{0} H=-0.1 \mathrm{~T}$ in Fig. 2(d)]: FMR absorption at a negatively swept magnetic field [positive $H$ in Figs. 2(b) and 2(d)] is substantially stronger than at a positively swept magnetic field [negative $H$ in Figs. 2(b) and 2(d)]. In addition, at $T<T_{c}$ a suppression of FMR response is observed in the low-field region $\left|\mu_{0} H\right|<0.02 \mathrm{~T}$. Below we discuss the FMR response of YIG in the absence of superconductivity, establish causes for the splitting of FMR at $\left|\mu_{0} H\right|<4 \pi M_{\text {eff, }}$ and define the contribution of superconductivity to the FMR spectrum.

\section{A. FMR at $T>T_{c}$ : Magnetic properties of YIG film at cryogenic temperatures}

Having analyzed possible origins for the splitting of the FMR into the $\mathrm{C}$ line and $\mathrm{G}$ line in Fig. 2, we can state that neither domain structure nor spin waves can contribute to the FMR spectrum for our particular study. For instance, nucleation of magnetic domains during demagnetization at $\mu_{0} H<4 \pi M_{\text {eff }}$ occurs for thin films with 
strong perpendicular anisotropy in comparison with the demagnetizing energy [36,37]; that is, when the magnetic quality parameter $Q=K_{u} / 2 \pi M_{s}^{2}>1$, where $K_{u}$ is the out-of-plane uniaxial anisotropy and $M_{S}$ is the saturation magnetization. However, a typical field of uniaxial anisotropy $\mu_{0} H_{K_{u}}=2 K_{u} / M_{s}$ in LPE-grown YIG thin films ranges up to approximately $200 \mathrm{Oe}[23,28,32,38]$, ensuring $Q \ll 1$. The highest values of uniaxial anisotropy in YIG films $\mu_{0} H_{K_{u}} \sim 10^{3}$ Oe that can be obtained in pulsed-laserdeposited films [39] still ensure $Q<1$. As an additional test, we perform a magnetic-force-microscopy study of the magnetic flux structure at the surface of the YIG film at $4 \mathrm{~K}$ using an attocube attoDRY1000 closed-cycle cryogenic microscope, supplied with a superconducting solenoid, and find no traces of domains or any field-dependent magnetic structure. Therefore, we confirm that formation of the domain structure does not occur. The magnetic state of the YIG film is a single domain, and variation of the out-of-plane component of the magnetization at $\mu_{0} H<$ $4 \pi M_{\text {eff }}$ occurs via rotation of the magnetization vector from out-of-plane orientation to in-plane orientation.

The absence of a contribution of standing-spin-wave resonances to the FMR spectrum can be illustrated in the following way. At $H=0$ the magnetization vector of a single-domain film is oriented in-plane. Therefore, the Kittel formula for the FMR and dispersion relations for any spin-wave mode at $H=0$ become applicable. When several resonances are observed, a contributing spinwave mode can be identified by estimating a resonance frequency difference $\Delta f_{r}$ between the Kittel mode and any standing-spin-wave mode. The latter appears due to quantization of the wavelength with geometrical parameters of a sample. The difference $\Delta f_{r}$ is then compared with the experimentally observed difference of approximately $1.3 \mathrm{GHz}$ at $H=0$ (Fig. 2). If an in-plane magnetostatic standing-spin-wave mode $[40,41]$ is assumed (e.g., the backward volume mode or the magnetostatic surface mode), its wavelength $\lambda / 2$ for the standing mode should be quantized with the dimensions of the CPW (i.e., $\lambda / 2 \sim 20-40 \mu \mathrm{m}$ ). Such standing mode provides only a marginal difference $\Delta f_{r} \lesssim 10 \mathrm{MHz}$ due to a small ratio $d / \lambda \sim 10^{-3}$. Alternatively, if exchange-dominated perpendicular standing-spin-wave resonance $[42,43]$ is assumed, the typical exchange constant in YIG films [32] (approximately $4 \times 10^{-12} \mathrm{~J} / \mathrm{m}$ ) provides $\Delta f_{r} \sim 2.5 \mathrm{GHz}$ for $d=\lambda / 4$ and $\Delta f_{r} \sim 7.5 \mathrm{GHz}$ for $d=\lambda / 2$. Thus, none of the possible standing-spin-wave modes can provide $\Delta f_{r} \sim 1.3 \mathrm{GHz}$. Overall, when a standing spin-wave resonance is excited, multiple consequential spectral lines are expected. FMR absorption for each line should decrease progressively with the mode number (see, e.g., Ref. [44]). Such a picture is not observed in our experiment. Therefore, we confirm that several spectral lines in Fig. 2 at $\left|\mu_{0} H\right|<4 \pi M_{s}$ are not caused by standing-spin-wave resonances.
The remaining explanation for two FMR lines requires the existence of two resonating areas with different magnetic properties in the vicinity of the CPW. The magnetic structure is essentially single-domain in each area. The resonating areas can be identified by the coupling strength of microwaves to precessing magnetization that is proportional to the FMR amplitude and correlates directly with the amplitude of excitation ac magnetic fields. In the CPW geometry, ac magnetic fields are mainly focused in the vicinity of the central transmission line $[12,25]$. Therefore, the geometry of the experiment (Fig. 1) suggests that the lower-frequency, weaker G line originates from YIG in gap areas of the CPW where the coupling is weaker, while the higher-frequency, stronger $\mathrm{C}$ line appears due to the FMR response of the YIG area under the central conducting line of the CPW. The accuracy of that explanation is strengthened by additional features, as discussed below. For the case of the single-domain single-crystal YIG film, the analytical resonance curve $f_{r}\left(\mu_{0} H\right)$ can be obtained in the entire $H$ range following Refs. [38,45-47] (we keep the notation given in Ref. [38]). The orientation of the magnetization of a single-domain film in an arbitrarily oriented magnetic field is defined by the minimum of the free magnetostatic energy $g=g\left(M_{s}, h, k_{1}, k_{2}, k_{u}, \theta, \psi, \phi_{h}, \phi_{m}\right)$, where $k_{1}$ and $k_{2}$ are unitless parameters of the cubic magnetocrystalline anisotropy, $k_{u}$ is a unitless parameter of the out-of-plane uniaxial anisotropy, $h=\mu_{0} H / 4 \pi M_{s}$ is the normalized external magnetic field, and $\theta, \psi, \phi_{h}$, and $\phi_{m}$ define the orientations of $H$ and $M_{s}$ with respect to the principal crystallographic axes of YIG in spherical coordinates (see Fig. 3). In addition, the system in Fig. 1 has a distinct directionality along the orientation of the CPW. This directionality may contribute to the orientation of magnetization. We account for its possible contribution by an additional energy term $g_{a}$ added to the free magnetostatic energy $g$ that provides a phenomenological in-plane uniaxial anisotropy of the first order. The term of the in-plane uniaxial anisotropy of the first order in the coordinates in Fig. 3 is

$$
g_{a}=-k_{a_{1}} \sin \psi^{2} \cos \left(\phi_{h}-\alpha\right)^{2} .
$$

The FMR frequency is defined by derivatives at the position of the minimum of the free energy [45-47] as

$$
f_{r} \sim \gamma\left(g_{\psi \psi} g_{\phi_{m} \phi_{m}}-g_{\psi \phi_{m}}^{2}\right)^{1 / 2} / \sin \psi,
$$

where $\gamma$ is the gyromagnetic ratio (see Refs. [38,45-47] for details). The dotted data points in Fig. 4(a) show the experimental $f_{r}\left(\mu_{0} H\right)$ resonance curves extracted from Fig. 2(a). First, we focus on the $\mathrm{G}$ line of the FMR spectrum. To fit the data, we develop the following routine, which allows us to obtain all magnetic parameters of $g$ and $f_{r}$, despite a large number of parameters and their partial interdependency. First, when misalignment $\theta$ of the orientation 


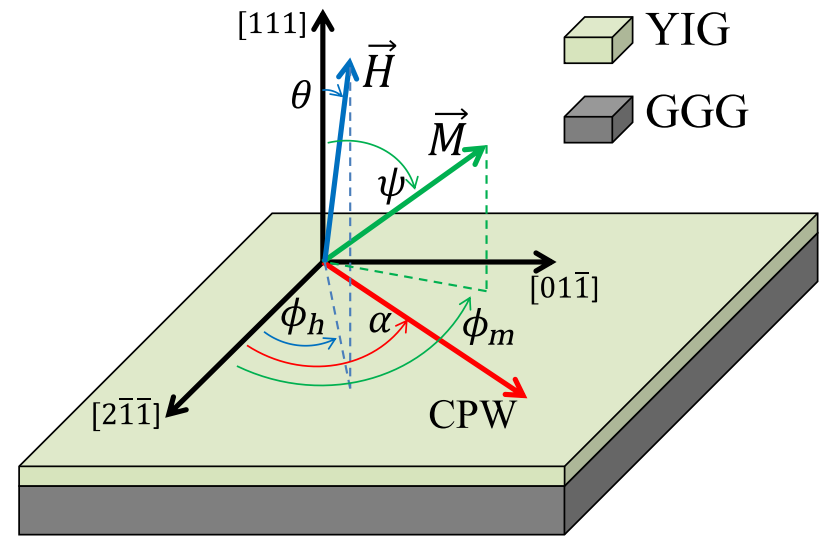

FIG. 3. Spherical coordinate system for the YIG-film sample. The direction of the CPW transmission line (see Fig. 1) is indicated by the red axis, which specifies additional in-plane uniaxial anisotropy due to CPW directionality.

of the magnetic field with the $z$ axis is small, and inplane CPW uniaxial anisotropy $k_{a_{1}}$ is negligible, the linear part at $\mu_{0} H \gg 4 \pi M_{\text {eff }}$ can be fitted with the simplified expression [38]

$$
f_{r}=2 \gamma M_{s}\left[h-\left(1-k_{u}+2 k_{1} / 3+2 k_{2} / 9\right)\right]
$$

Fitting the data in the field range from 0.3 to $0.4 \mathrm{~T}$ with Eq. (3), we obtain the gyromagnetic ratio $\gamma / 2 \pi=$ $2.985 \mathrm{GHz} / \mathrm{kOe}$, which is close to the value for a free electron, $2.803 \mathrm{GHz} / \mathrm{kOe}$, and also the saturation magnetization in relation to anisotropy parameters, $4 \pi M_{\text {eff }}=$ $4 \pi M_{s} \times\left(1-k_{u}+2 k_{1} / 3+2 k_{2} / 9\right)=1975$ Oe. Next we note that (i) the position of the kink at $\mu_{0} H \simeq 0.22 \mathrm{~T}$ in the $f_{r}$-versus- $\mu_{0} H$ plot is mostly determined by misalignment of the magnetic field with the $z$ axis (i.e., by $\theta$ and $\phi_{h}$ ), (ii) the position of the maximum of the FMR frequency at $\mu_{0} H \simeq 0.12 \mathrm{~T}$ in the $f_{r}$-versus- $\mu_{0} H$ plot is mostly determined by the magnetocrystalline-anisotropy parameters $k_{1}$ and $k_{2}$, and (iii) the slope of the resonance curve $f_{r}\left(\mu_{0} H\right)$ at $H \rightarrow 0$ and the value $f_{r}(H=0)$ are defined by the CPW-induced uniaxial anisotropy [i.e., by $k_{a_{1}}$ and $\alpha$ in Eq. (1)]. Using the least-squares method for optimization through steps (i) to (iii) and back, after several runs, (iv) the fit is further optimized by our adjusting the value of the parameter $k_{u}$. Following steps (i)-(iv) we obtain an optimum fit of the G line [see the red curve in Fig. 4(a)] with the following parameters: $4 \pi M_{s}=1876$ Oe, $k_{1}=-0.16$, $k_{2}=0.18, k_{u}=-0.12, \theta=1.4, \phi_{h}=126, k_{a_{1}}=0.025$, and $\alpha=177$. Importantly, the values of the parameters of the cubic magnetocrystalline anisotropy $k_{1}$ and $k_{2}$ are a factor of 2-3 higher than typical values at room temperature $[23,38]$. This trend correlates well with the temperature dependence of the cubic magnetocrystalline anisotropy in YIG bulk single crystals [48].

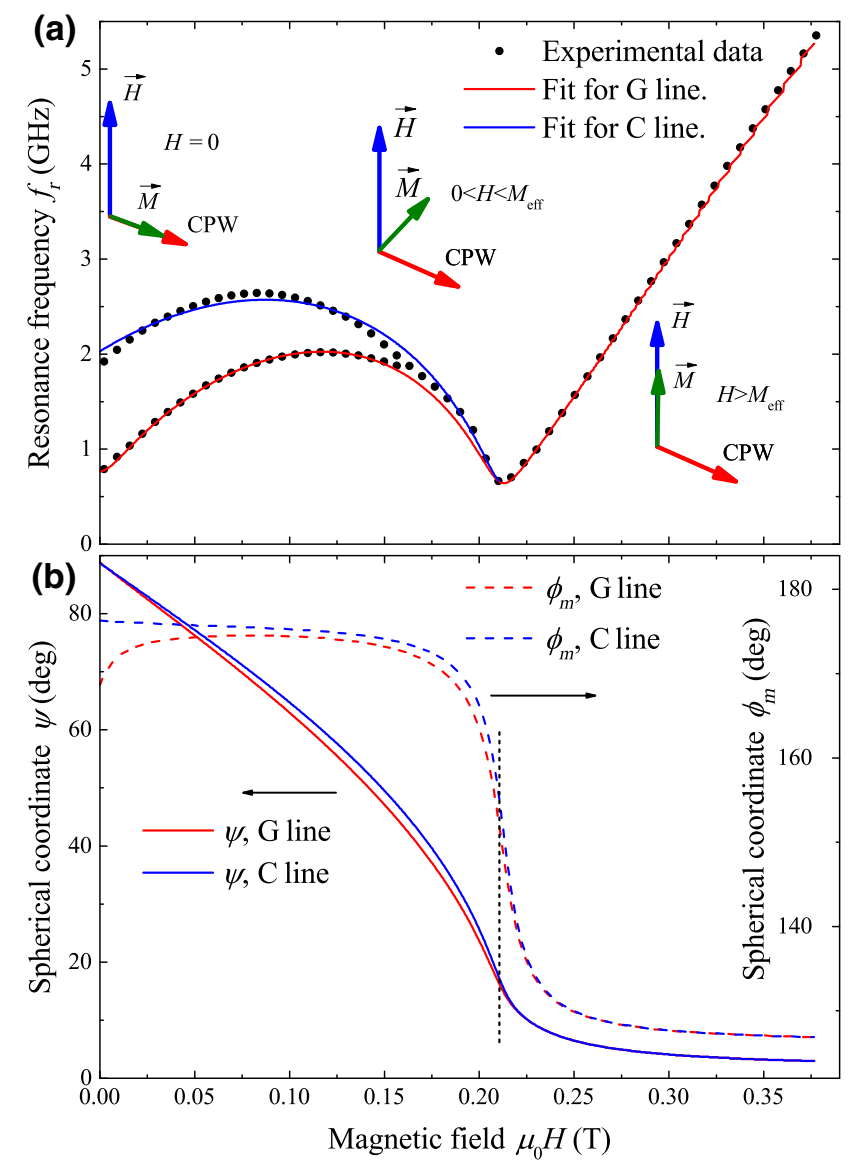

FIG. 4. Fitting experimental $f_{r}\left(\mu_{0} H\right)$. (a) The dependence of the FMR frequencies on magnetic field. The CPW induces uniaxial anisotropy of the first order ( $\mathrm{G}$ line) and the second order $(\mathrm{C}$ line). The pictograms in (a) illustrate the orientation of the magnetization relative to the orientations of the magnetic field and the CPW axis at different magnetic fields. (b) The dependence of free-energy-minimum orientations of the magnetization on the magnetic field. The dotted black line indicates the position of the kink in the FMR curves.

After fitting the $\mathrm{G}$ line, which corresponds to the FMR response of YIG areas in the CPW gaps, our only option to fit the $\mathrm{C}$ line, which corresponds to the FMR response of YIG areas under the central CPW line, is to introduce an additional term into the energy $g$ that represents the second-order uniaxial anisotropy induced by the CPW. The term of the in-plane uniaxial anisotropy of the second order in the coordinates in Fig. 3 is

$$
\begin{aligned}
g_{a}= & -\left(k_{a_{1}}+2 k_{a_{2}}\right) \sin \psi^{2} \cos \left(\phi_{h}-\alpha\right)^{2} \\
& +k_{a_{2}} \sin \psi^{4} \cos \left(\phi_{h}-\alpha\right)^{4}
\end{aligned}
$$

With use of magnetic parameters obtained for the G line, the fitting procedure for the $\mathrm{C}$ line with the anisotropy given by Eq. (4) provides $k_{a_{1}}=0.121$ and $k_{a_{2}}=-0.048$. This fit is shown in Fig. 4(a) as the blue curve. 
Possible origins of the CPW-induced anisotropy include a distinct directionality of microwave currents. Also, directionality of the surface stress can be considered that appears from differences in thermal expansion of the narrow central transmission line of metal CPW and YIG/GGG oxides. The surface stress may appear either due to deposition of $\mathrm{Nb}$ film at elevated temperature or due to performance of experiments at cryogenic temperatures. For instance, the difference in thermal expansions between $\mathrm{Nb}$ and garnets can produce a strain $\epsilon$ in YIG at $2 \mathrm{~K}$ of up to approximately $6 \times 10^{-4}$ along the CPW in the absence of mechanical relaxation in $\mathrm{Nb}$. In contrast, if complete relaxation of tensions occurs in $\mathrm{Nb}$ at room temperature, the difference in thermal expansions produces the opposite-sign strain in YIG of approximately $-4 \times 10^{-4}$. Both values of the strain are well comparable with the growth-induced tensions provided by the lattice misfit between the GGG substrate and the YIG film that induces the uniaxial anisotropy in LPE-grown films [28] and films grown by pulsed-laser deposition [49,50] (see the Appendix for details). Importantly, the presence of both first- and second-order anisotropies suggests different mechanisms for their induction.

Figure 4(b) shows the dependence of the orientation of magnetization $\psi\left(\mu_{0} H\right)$ and $\phi_{m}\left(\mu_{0} H\right)$ for $\mathrm{C}$ and G FMR lines on the magnetic field. A marginal difference between the $C$ and $G$ curves in the entire field range indicates coalignment of magnetization orientations in both gap and center areas of the CPW, implying that the entire volume of YIG that is subjected to the FMR remains in the single-domain state throughout the experiment.

Our experimental setup does not allow us to study microwave transmission at higher temperatures $T \gtrsim 15 \mathrm{~K}$. Therefore, the temperature dependence of the magnetic parameters of YIG is not addressed here, and can be found elsewhere $[22,23,51]$.

\section{B. FMR at $T<T_{c}$ : Impact of the superconducting critical state}

At $T<T_{c}$ of $\mathrm{Nb}$, in the presence of superconductivity, the FMR absorption spectrum changes [see Fig. 2(b)]. Since the Nb CPW is placed directly on top of the YIG film, all changes in absorptions in the $\mathrm{C}$ branch can be attributed to the magnetization state under the $\mathrm{Nb}$ line. Therefore, the effect of superconductivity on the FMR can be tracked analyzing the superconducting critical state of $\mathrm{Nb}$ film and its variation with applied magnetic field.

Figure 5(a) shows the zero-field-cooled (ZFC) transmission spectrum that is acquired when the sample is cooled to $2 \mathrm{~K}$ at zero magnetic field, and afterward $S_{21}$ measurements are performed while the magnetic field is swept from 0 to 0.11 T. Figure 5(b) shows the field-cooled (FC) transmission spectrum that is acquired when the sample is cooled to $2 \mathrm{~K}$ at $\mu_{0} H=0.25 \mathrm{~T}$, and afterward $S_{21}$ measurements are performed while the magnetic field is swept from 0.11 to $0 \mathrm{~T}$. The hysteresis in peak absorption is tracked by our fitting $S_{21}(f)$ curves at each value of $H$ and plotting the dependence of the FMR amplitude $I$ on the magnetic field $H$ [Fig. 5(c)]. $I\left(\mu_{0} H\right)$ dependency is caused by variation of the CPW FMR coupling strength with magnetic field (i.e., by variation of magnetization and magnetic flux inhomogeneity in the YIG induced by the $\mathrm{Nb}$ superconducting critical state). Importantly, no hysteresis in peak absorption is observed at $T>T_{c}$ [Figs. 2(a) and 5(c)], where the transmission spectrum is fully reversible and independent of the ZFC or FC initial state.

First we discuss the ZFC curve in Fig. 5(c), where three intervals in $I\left(\mu_{0} H\right)$ can be distinguished. At low fields, the strongest FMR absorption is observed with $I \sim 0.1$ at $\mu_{0} H$ up to $2 \times 10^{-3} \mathrm{~T}$ [highlighted by the red circle in Fig. 5(a)]. This corresponds to the Meissner state of the $\mathrm{Nb}$ line when the Meissner screening currents circulate at the edges of the $\mathrm{Nb}$ film and exclude magnetic flux from its cross section. In the Meissner state, dc magnetic flux remains homogeneous across the $\mathrm{Nb}$ line and ensures a strong coupling of the CPW to the YIG at FMR. At intermediate fields, $2 \times 10^{-3}<\mu_{0} H<10^{-2} \mathrm{~T}$, the FMR absorption drops rapidly from $I \sim 0.1$ to the minimum $I \sim 0.02$, caused by the partially penetrated superconducting critical state where superconducting vortices start to penetrate $\mathrm{Nb}$ film. The magnetic flux profile in partially penetrated superconducting films is the most inhomogeneous [52-54], which causes a weak coupling of the FMR to the CPW and low absorption intensity. The partially penetrated state commences at the flux-focus enhanced first critical field of the superconducting film $\mu_{0} H_{c_{1}} \sim 2 \times 10^{-3} \mathrm{~T}$, where the first Abrikosov vortices start to penetrate into the film, and terminates at the magnetic field of full penetration, $10^{-2} \mathrm{~T}$. At high fields, $\mu_{0} H>0.01 \mathrm{~T}$, after full penetration is reached, the magnetic flux in the superconducting film forms a constant gradient that can be depicted by the Bean critical state model [55-58]. The gradient is formed due to pinning of vortices and induces a homogeneous circulating critical currents. On increase of the magnetic field, both the pinning of vortices and the slope of the magnetic flux reduce [57,58], making the magnetic flux in YIG more homogeneous. A smaller gradient of the magnetic flux in the superconductor increases the coupling that we observe in a gradual increase of the FMR peak absorption on increase of the magnetic field from 0.01 $\mathrm{T}$ to higher fields. Note that such nonmonotonic behavior of $I\left(\mu_{0} H\right)$ is not observed for the G line [Fig. 5(a)], which indicates additionally that the absorption at the $G$ line is caused by the FMR in the gap areas of the CPW, where the influence of the superconducting state of $\mathrm{Nb}$ is marginal.

On increase of the magnetic field further beyond the field range in Fig. 5, the ZFC curve should coincide with the FC curve at the so-called irreversibility field 

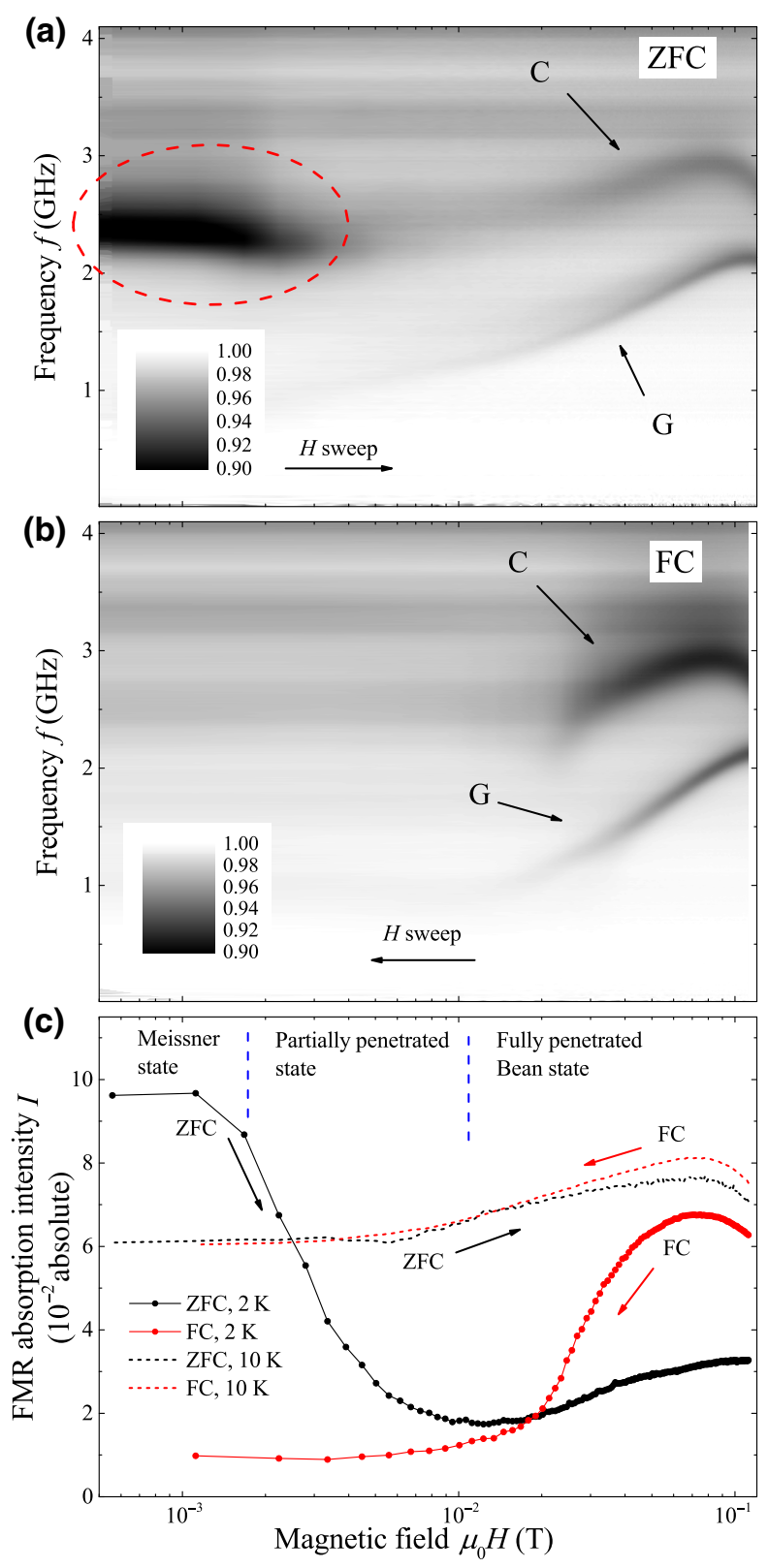

FIG. 5. Gray-scale-coded transmission spectra $\left|S_{21}\left(\mu_{0} H, f\right) / S_{21}\left(\mu_{0} H=0.5 \mathrm{~T}, f\right)\right|$ measured at $2 \mathrm{~K}$ starting from the ZFC state (a) and the FC state (b). C and G spectral lines are indicated. The red circle in (a) highlights FMR absorption in the Meissner state of the CPW. (c) Dependencies of resonance peak absorption for the $\mathrm{C}$ line on magnetic field $I\left(\mu_{0} H\right)$ obtained at 2 and $10 \mathrm{~K}$. The direction of magnetic field sweep is indicated by arrows. The $\mu_{0} H$ axis is given on a log scale. The field regions for three superconducting states of $\mathrm{Nb}$ in the ZFC curve in (c) are separated by dashed blue lines.

[58-60], where pinning of vortices becomes negligible. The FC curve in Fig. 5 consists of two parts. For $\mu_{0} H>$ $0.03 \mathrm{~T}$, the coupling remains a factor of approximately 2 greater than that for the ZFC curve. This difference is attributed to the fact that on decease of the magnetic field, the Bean critical currents counteract the Meissner currents, diamagnetic response of the superconducting film is reduced as compared with the ZFC measurement [60], and the influence on YIG at the FMR decreases. Below $0.03 \mathrm{~T}, I$ drops rapidly, which can be explained by the gradual formation of a complex remanent critical state at $H=0$ with highly nonuniformly distributed frozen magnetic flux. Also, at low magnetic fields, magnetization of individual Abrikosov vortices may contribute to YIG inhomogeneity by inducing substantial local magnetic fields of up to $\mu_{0} H_{v} \sim \Phi_{0} / \pi \lambda_{L}^{2} \sim 0.06 \mathrm{~T}$, where $\Phi_{0}$ is the magnetic flux quantum and $\lambda_{L} \sim 10^{-7} \mathrm{~m}$ is the typical London penetration depth in $\mathrm{Nb}$ films.

Overall, the influence of the superconducting critical state in our geometry on the FMR appears to be destructive. The FMR intensity for the ZFC and FC curves remains below the values of $I$ at $T>T_{c}$ [Fig. 5(c)]. However, magnetic hysteresis is often used in magnetic logic devices. Also, in vicinity to $H=0$, FMR is substantially stronger when the superconductor is in the Meissner state than for a normal-metal CPW. This effect may be a result of interaction of magnetic moments in YIG with Meissner screening currents in the ideal diamagnet.

\section{CONCLUSION}

In conclusion, ferromagnetic resonance of YIG film is studied in out-of-plane magnetic fields and at cryogenic temperatures with a superconducting coplanar waveguide that is fabricated directly on top of the magnetic film (see Fig. 1). FMR absorption spectra are obtained in a wide field range. Nonlinear dependence of the FMR frequency on magnetic field at low field values, below the field of saturation magnetization, shows a split of the resonance into two spectral lines, which are identified as the FMR response of YIG in gap areas of the CPW and of YIG located directly under the central conducting line of the CPW.

A routine is developed for fitting the FMR lines. This routine allows us to obtain all magnetic parameters of YIG (i.e., the saturation magnetization, the gyromagnetic ratio, and parameters of magnetocrystalline and out-of-plane uniaxial anisotropies). In addition, the fitting routine gives a misalignment angle of $1.4^{\circ}$ between the magnetic field and the out-of-plane orientation, as well as parameters of the in-plane magnetic anisotropy of the first and the second order, which are induced by the CPW.

The FMR spectrum at temperatures below the superconducting critical temperature of the waveguide shows a hysteresis in FMR peak absorption. The hysteresis is explained by the influence of magnetization of the $\mathrm{Nb}$ transmission line in the superconducting critical state. Tracking the dependence of the intensity of the FMR on the magnetic field allows us to identify all fundamental states 
of a superconducting film in an out-of-plane magnetic field (i.e., the Meissner state, the partially penetrated state, and the fully penetrated Bean critical state). Also, it allows us to explain the hysteresis in the FMR absorption by the pinning of magnetic vortices, which induces the gradient of magnetic flux in superconducting films. The gradient is controlled by the direction of the magnetic field sweep.

In general, we suggest that development of magnonics at cryogenic temperatures may be beneficial due to (i) substantially different properties of magnetic materials, including magnetocrystalline anisotropy, (ii) the possibility to engineer additional anisotropies with metal structures, and (iii) the potential to affect the spectra by hybridization of a magnonic medium with superconductors. As a final remark, we mention related work by Jeon et al. [61] on the effect of the superconducting critical state on magnetization dynamics in thick superconductor/ferromagnet/superconductor trilayers.

\section{ACKNOWLEDGMENTS}

The authors acknowledge Lucas Radtke and Yannick Schoen for assistance with sample preparation and initial measurements and Paul Baity for critical reading of the manuscript. This work was supported by the European Research Council under the Grant Agreement No. 648011 and the Deutsche Forschungsgemeinschaft (DFG) within the project INST 121384/138-1 FUGG. C.D. thanks the DFG for financial support under Contract No. DFG DU 1427/2-1. I.A.G. acknowledges support by the German Academic Exchange Service (DAAD) via the program "Research Stays for University Academics and Scientists 2017." I.A.G., N.N.A., V.V.R., and A.V.U. acknowledge the Ministry of Education and Science of the Russian Federation (Research Project K2-2018-015 in the framework of the Increase Competitiveness Program of NUST "MISiS") for support with microwave measurements. V.S.S., I.A.G., and D.S.B. acknowledge the Russian Science Foundation (Project No. 18-72-10118) for support with numerical analysis and magnetic force microscopy investigations. V.V.R. acknowledges partial support by the Russian Foundation for Basic Research (Project No. 19-02-00316). A.A.G. acknowledges partial support by the EU H2020-WIDESPREAD-05-2017-Twinning project "SPINTECH" under the Grant Agreement No. 810144.

\section{APPENDIX: STRESS INDUCED IN YIG BY THE Nb CPW}

One possible cause of the CPW-induced anisotropy that is derived in Sec. III A is the stress in YIG that is caused by differences in thermal expansion of the narrow extended central transmission line of the metal CPW and YIG/GGG oxides. Assuming that an unstressed continuous interface is formed between $\mathrm{Nb}$ and YIG during deposition of $\mathrm{Nb}$ at the deposition temperature $T_{d} \approx 600 \mathrm{~K}$, the stress at the interface at the measurement temperature $T_{m}=2 \mathrm{~K}$ can be estimated with the following expression:

$$
\sigma \approx \frac{E}{1-v} \epsilon=\frac{E}{1-v} \int_{T_{d}}^{T_{m}}\left[\alpha_{G}(T)-\alpha_{\mathrm{Nb}}(T)\right] d T,
$$

where $\sigma$ is the stress in YIG, $E=2 \times 10^{12} \mathrm{dyn} / \mathrm{cm}^{2}$ is the Young's modulus of YIG in the temperature range from 0 to $300 \mathrm{~K}$ [62], $v=0.29$ is the Poisson's ratio, $\epsilon$ is the strain at the interface at $T_{m}$ due to the difference in thermal expansion, and $\alpha_{G}(T)$ and $\alpha_{\mathrm{Nb}}(T)$ are the temperature dependencies of the linear thermal expansion of garnet and $\mathrm{Nb}$, respectively. Importantly, the stress in Eq. (A1) implies the absence of mechanical relaxation.

However, estimation of the stress at the $\mathrm{Nb} / \mathrm{YIG}$ interface using Eq. (A1) is impeded. While the thermomechanical properties of $\mathrm{Nb}$ have been well studied in a wide temperature range [63] from approximately $0 \mathrm{~K}$ up to about the melting point, a consistent study of the thermomechanical properties of YIG is not available for the required temperature range. The coefficient $\alpha_{G}(T)$ for YIG is available piecewise and can be obtained by interpolation of $\alpha_{G}(T)$ at temperatures above $[64,65]$ and below [66] room temperature. On the other hand, the coefficient $\alpha_{G}(T)$ for YIG can be replaced with one for GGG since their thermomechanical properties are almost identical $[64,65]$. The coefficient $\alpha_{G}(T)$ for GGG has been reported for several temperature ranges separately: room-temperature and higher-temperature data are available in Refs. [64,65], $\alpha_{G}(T)$ at low temperatures is reported in Ref. [67] for the range from 6 to $300 \mathrm{~K}$ and in Ref. [68] for the range from 80 to $330 \mathrm{~K}$.

Figure 6 shows dependence of the thermal expansion on temperature $\alpha(T)$. The red curve shows $\alpha_{\mathrm{Nb}}(T)$ for $\mathrm{Nb}$ that is calculated with data from Ref. [63]. The blue curve shows $\alpha_{G}(T)$ for YIG that is calculated with data from Refs. [64,66]. The dashed and dotted black curves

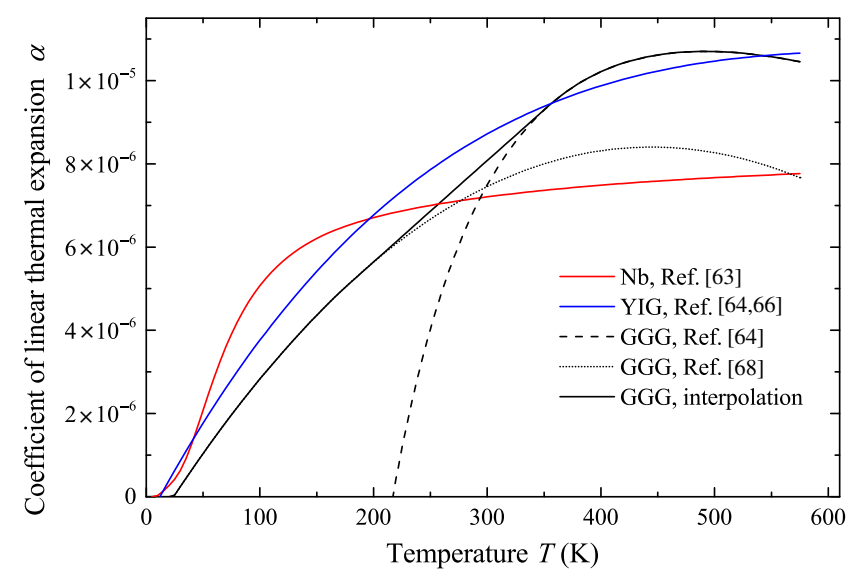

FIG. 6. Dependence of the thermal expansion coefficient on temperature $\alpha(T)$ for $\mathrm{Nb}$, YIG, and GGG. 
show $\alpha_{G}(T)$ for GGG that are calculated with data from Refs. [64,68]. The solid black curve shows linear interpolation between lower-temperature and higher-temperature curves $\alpha_{G}(T)$ for GGG in the range from 180 to $330 \mathrm{~K}$. The interpolated dependence for $\alpha_{G}(T)$ is used for the calculations.

Calculations with Eq. (A1) and the coefficients $\alpha(T)$ in Fig. 6 provide the strain at the $\mathrm{YIG} / \mathrm{Nb}$ interface, $\epsilon \approx 6.4 \times 10^{-4}$, that produces a compressive stress, $\sigma \sim$ $10^{9} \mathrm{dyn} / \mathrm{cm}^{2}$. Note, however, that if room-temperature deposition of $\mathrm{Nb}$ occurs, or the strain in $\mathrm{Nb}$ relaxes at room temperature, according to Eq. (A1) and Fig. 6 an opposite-sign strain, $\epsilon \approx-4 \times 10^{-5}$, emerges at cryogenic temperature $T_{m}$. If the data for GGG are used instead of the data for YIG, the integral in Eq. (A1) provides approximately the same strain, $\epsilon \approx 5.6 \times 10^{-4}$, at the interface with unrelaxed $\mathrm{Nb}$, and a larger opposite-sign strain, $\epsilon \approx$ $-4 \times 10^{-4}$, at the interface with the room-temperaturedeposited or room-temperature-relaxed $\mathrm{Nb}$. These values are comparable with the growth-induced tensions provided by the lattice misfit between the GGG substrate and the YIG film that induces the uniaxial anisotropy in LPE-grown films [28] and films grown by pulsed-laser deposition $[49,50]$.

[1] B. Lenk, H. Ulrichs, F. Garbs, and M. Münzenberg, The building blocks of magnonics, Phys. Rep. 507, 107 (2011).

[2] A. V. Chumak, V. I. Vasyuchka, A. A. Serga, and B. Hillebrands, Magnon spintronics, Nat. Phys. 11, 453 (2015).

[3] Y. Kajiwara, K. Harii, S. Takahashi, J. Ohe, K. Uchida, M. Mizuguchi, H. Umezawa, H. Kawai, K. Ando, K. Takanashi, S. Maekawa, and E. Saitoh, Transmission of electrical signals by spin-wave interconversion in a magnetic insulator, Nature 464, 262 (2010).

[4] Special Issue on Magnonics, J. Phys. D: Appl. Phys. 50 (2017).

[5] S. O. Demokritov and A. N. Slavin, eds. Magnonics: From fundamentals to applications (Springer-Verlag Berlin Heidelberg, 2013).

[6] M. Evelt, L. Soumah, A. B. Rinkevich, S. O. Demokritov, A. Anane, V. Cros, Jamal Ben Youssef, G. de Loubens, O. Klein, P. Bortolotti, and V. E. Demidov, Emission of Coherent Propagating Magnons by Insulator-Based SpinOrbit-Torque Oscillators, Phys. Rev. Appl. 10, 041002 (2018).

[7] H. Huebl, C. W. Zollitsch, J. Lotze, F. Hocke, M. Greifenstein, A. Marx, R. Gross, and S. T. B. Goennenwein, High Cooperativity in Coupled Microwave Resonator Ferrimagnetic Insulator Hybrids, Phys. Rev. Lett. 111, 127003 (2013).

[8] Y. Tabuchi, S. Ishino, T. Ishikawa, R. Yamazaki, K. Usami, and Y. Nakamura, Hybridizing Ferromagnetic Magnons and Microwave Photons in the Quantum Limit, Phys. Rev. Lett. 113, 083603 (2014).

[9] X. Zhang, C.-L. Zou, L. Jiang, and H. X. Tang, Strongly Coupled Magnons and Cavity Microwave Photons, Phys. Rev. Lett. 113, 156401 (2014).
[10] R. G. E. Morris, A. F. van Loo, S. Kosen, and A. D. Karenowska, Strong coupling of magnons in a YIG sphere to photons in a planar superconducting resonator in the quantum limit, Sci. Rep. 7, 11511 (2017).

[11] Marco Pfirrmann, Isabella Boventer, Andre Schneider, Tim Wolzand Mathias Kläui, Alexey V. Ustinov, and Martin Weides, Magnons at low excitations: Observation of incoherent coupling to a bath of two-level-systems, arXiv:1903.03981, (2019).

[12] I. A. Golovchanskiy, N. N. Abramov, V. S. Stolyarov, I. V. Shchetinin, P. S. Dzhumaev, A. S. Averkin, S. N. Kozlov, A. A. Golubov, V. V. Ryazanov, and A. V. Ustinov, Probing dynamics of micro-magnets with multi-mode superconducting resonator, J. Appl. Phys. 123, 173904 (2018).

[13] S. E. Barnes, M. Aprili, I. Petkovic, and S. Maekawa, Ferromagnetic resonance with a magnetic Josephson junction, Supercond. Sci. Technol. 24, 024020 (2011).

[14] S. Mai, E. Kandelaki, A. F. Volkov, and K. B. Efetov, Interaction of Josephson and magnetic oscillations in Josephson tunnel junctions with a ferromagnetic layer, Phys. Rev. B 84, 144519 (2011).

[15] I. A. Golovchanskiy, N. N. Abramov, V. S. Stolyarov, O. V. Emelyanova, A. A. Golubov, A. V. Ustinov, and V. V. Ryazanov, Ferromagnetic resonance with long Josephson junction, Supercond. Sci. Technol. 30, 054005 (2017).

[16] B. M. Lebed and S. V. Yzkovlev, Dispersion of surface spin waves in a layered superconductor-ferrite structure, Pis'ma v ZhTF (in Russian) 15, 27 (1989).

[17] V. B. Anfinogenov, Y. V. Gulyaev, P. E. Zilberman, I. M. Kotelyanskiy, N. I. Polzikova, and A. A. Suhanov, Observation of the electronic absorption of magnetostatic waves in a ferrite-high temperature superconductor structure, Pis'ma v ZhTF (in Russian) 15, 24 (1989).

[18] I. A. Golovchanskiy, N. N. Abramov, V. S. Stolyarov, V. V. Bolginov, V. V. Ryazanov, A. A. Golubov, and A. V. Ustinov, Ferromagnet/superconductor hybridization for magnonic applications, Adv. Funct. Mater. 28, 1802375 (2018).

[19] I. A. Golovchanskiy, N. N. Abramov, V. S. Stolyarov, V. V. Ryazanov, A. A. Golubov, and A. V. Ustinov, Modified dispersion law for spin waves coupled to a superconductor, J. Appl. Phys. 124, 233903 (2018).

[20] O. V. Dobrovolskiy, R. Sachser, T. Brächer, T. Böttcher, V. V. Kruglyak, R. V. Vovk, V. A. Shklovskij, M. Huth, B. Hillebrands, and A. V. Chumak, Magnon-fluxon interaction in a ferromagnet/superconductor heterostructure, Nat. Phys. (2019).

[21] A. Pimenov, A. Loidl, P. Przyslupski, and B. Dabrowski, Negative Refraction in Ferromagnet-Superconductor Superlattices, Phys. Rev. Lett. 95, 247009 (2005).

[22] M. Haidar, M. Ranjbar, M. Balinsky, R. K. Dumas, S. Khartsev, and J. Akerman, Thickness- and temperaturedependent magnetodynamic properties of yttrium iron garnet thin films, J. Appl. Phys. 117, 17D119 (2015).

[23] N. Beaulieu, N. Kervarec, N. Thiery, O. Klein, V. Naletov, H. Hurdequint, G. de Loubens, J. B. Youssef, and N. Vukadinovic, Temperature dependence of magnetic properties of a ultrathin yttrium-iron garnet film grown by liquid phase epitaxy: Effect of a Pt overlayer, IEEE Magn. Lett. 9, 3706005 (2018). 
[24] I. Boventer, M. Pfirrmann, J. Krause, Y. Schön, M. Kläui, and $\mathrm{M}$. Weides, Complex temperature dependence of coupling and dissipation of cavity magnon polaritons from millikelvin to room temperature, Phys. Rev. B 97, 184420 (2018).

[25] I. Neudecker, G. Woltersdorf, B. Heinrich, T. Okuno, G. Gubbiotti, and C. H. Back, Comparison of frequency, field, and time domain ferromagnetic resonance methods, J. Magn. Magn. Mat. 307, 148 (2006).

[26] S. S. Kalarickal, P. Krivosik, M. Wu, C. E. Patton, M. L. Schneider, P. Kabos, T. J. Silva, and J. P. Nibarger, Ferromagnetic resonance linewidth in metallic thin films: Comparison of measurement methods, J. Appl. Phys. 99, 093909 (2006).

[27] Y.-C. Chen, D.-S. Hung, Y.-D. Yao, S.-F. Lee, H.-P. Ji, and $\mathrm{C}$. $\mathrm{Yu}$, Ferromagnetic resonance study of thicknessdependent magnetization precession in $\mathrm{Ni}_{80} \mathrm{Fe}_{20}$ films, $\mathrm{J}$. Appl. Phys. 101, 09C104 (2007).

[28] C. Dubs, O. Surzhenko, R. Linke, A. Danilewsky, U. Brückner, Jan Dellith, Sub-micrometer yttrium iron garnet LPE films with low ferromagnetic resonance losses, J. Phys. D: Appl. Phys. 50, 204005 (2017).

[29] I. A. Golovchanskiy, V. V. Bolginov, N. N. Abramov, V. S. Stolyarov, A. BenHamida, V. I. Chichkov, D. Roditchev, and V. V. Ryazanov, Magnetization dynamics in dilute $\mathrm{Pd}_{1-x} \mathrm{Fe}_{x}$ thin films and patterned microstructures considered for superconducting electronics, J. Appl. Phys. 120, 163902 (2016).

[30] C. Kittel, On the theory of ferromagnetic resonance absorption, Phys. Rev. 73, 155 (1948).

[31] Y. V. Khivintsev, L. Reisman, J. Lovejoy, R. Adam, C. M. Schneider, R. E. Camley, and Z. J. Celinski, Spin wave resonance excitation in ferromagnetic films using planar waveguide structures, J. Appl. Phys. 108, 023907 (2010).

[32] S. Klingler, A. V. Chumak, T. Mewes, B. Khodadadi, C. Mewes, C. Dubs, O. Surzhenko, B. Hillebrands, and A. Conca, Measurements of the exchange stiffness of YIG films using broadband ferromagnetic resonance techniques, J. Phys. D: Appl. Phys. 48, 015001 (2015).

[33] J. O. Artman and S. H. Charap, Ferromagnetic resonance in periodic domain structures, J. Appl. Phys. 49, 1587 (1978).

[34] M. Ramesh and P. E. Wigen, Ferromagnetodynamics of parallel stripe domains - domain walls system, J. Mag. Mag. Mater. 74, 123 (1988).

[35] I. S. Camara, S. Tacchi, L.-C. Garnier, M. Eddrief, F. Fortuna, G. Carlotti, and M. Marangolo, Magnetization dynamics of weak stripe domains in $\mathrm{Fe}-\mathrm{N}$ thin films: A multi-technique complementary approach, J. Phys.: Condens. Matter. 29, 465803 (2017).

[36] T. G. W. Blake, C.-C. Shir, Y.-O. Tu, and E. D. Torre, Effects of finite anisotropy parameter $\mathrm{Q}$ in the determination of magnetic bubble material parameters, IEEE Trans. Magn. 18, 985 (1982).

[37] F. Virot, L. Favre, R. Hayn, and M. D. Kuz'min, Theory of magnetic domains in uniaxial thin films, J. Phys. D: Appl. Phys. 45, 405003 (2012).

[38] S. Lee, S. Grudichak, J. Sklenar, C. C. Tsai, M. Jang, Q. Yang, H. Zhang, and J. B. Ketterson, Ferromagnetic resonance of a YIG film in the low frequency regime, J. Appl. Phys. 120, 033905 (2016).
[39] S. A. Manuilov, S. I. Khartsev, and A. M. Grishin, Pulsed laser deposited $\mathrm{Y}_{3} \mathrm{Fe}_{5} \mathrm{O}_{12}$ films: Nature of magnetic anisotropy I, J. Appl. Phys. 106, 123917 (2009).

[40] D. Stancil, Theory of magnetostatic waves (Springer-Verlag New York, Inc., 1993).

[41] A. A. Serga, A. V. Chumak, and B. Hillebrands, YIG magnonics, J. Phys. D: Appl. Phys. 43, 264002 (2010).

[42] C. Kittel, Excitation of spin waves in a ferromagnet by a uniform rf field, Phys. Rev. 100, 1295 (1958).

[43] M. H. Seavey and P. E. Tannenwald, Direct observation of spin wave resonance, J. Appl. Phys. 30, S227 (1959).

[44] S. A. Bunyaev, V. O. Golub, O. Yu. Salyuk, E. V. Tartakovskaya, N. M. Santos, A. A. Timopheev, N. A. Sobolev, A. A. Serga, A. V. Chumak, B. Hillebrands, and G. N. Kakazei, Splitting of standing spin-wave modes in circular submicron ferromagnetic dot under axial symmetry violation, Sci. Rep. 5, 18480 (2015).

[45] J. Smit and H. G. Beljers, Ferromagnetic resonance absorption in $\mathrm{BaFe}_{12} \mathrm{O}_{19}$ highly anisotropic crystal, Philips Res. Rep. 10, 113 (1955).

[46] H. Suhl, Ferromagnetic resonance in nickel ferrite between one and two kilomegacycles, Phys. Rev. 97, 555 (1955).

[47] S. M. Rezende, J. A. S. Moura, F. M. de Aguiar, and W. H. Schreiner, Ferromagnetic resonance of $\mathrm{Fe}\left(\begin{array}{lll}1 & 1 & 1\end{array}\right)$ thin films and $\mathrm{Fe}\left(\begin{array}{lll}1 & 1 & 1\end{array}\right) / \mathrm{Cu}\left(\begin{array}{lll}1 & 1 & 1\end{array}\right)$ multilayers, Phys. Rev. B 49, 15105 (1994).

[48] P. Hansen, Anisotropy and magnetostriction of galliumsubstituted yttrium iron garnet, J. Appl. Phys. 45, 3638 (1974).

[49] Brandon M. Howe, Satoru Emori, Hyung-Min Jeon, Trevor M. Oxholm, John G. Jones, Krishnamurthy Mahalingam, Yan Zhuang, Nian X. Sun, and Gail J. Brown, Pseudomorphic yttrium iron garnet thin films with low damping and inhomogeneous linewidth broadening, IEEE Magn. Lett. 6, 3500504 (2015).

[50] Biswanath Bhoi, Bosung Kim, Yongsub Kim, Min-Kwan Kim, Jae-Hyeok Lee, and Sang-Koog Kim, Stress-induced magnetic properties of PLD-grown high-quality ultrathin YIG films, J. Appl. Phys. 123, 203902 (2018).

[51] H. Maier-Flaig, S. Klingler, C. Dubs, O. Surzhenko, R. Gross, M. Weiler, H. Huebl, and S. T. B. Goennenwein, Temperature-dependent magnetic damping of yttrium iron garnet spheres, Phys. Rev. B 95, 214423 (2017).

[52] Ch. Jooss, J. Albrecht, H. Kuhn, S. Leonhardt, and H. Kronmüller, Magneto-optical studies of current distributions in high- $T_{C}$ superconductors, Rep. Prog. Phys. 65, 651 (2001).

[53] F. S. Wells, A. V. Pan, S. Wilson, I. A. Golovchanskiy, S. A. Fedoseev, and A. Rozenfeld, Dynamic magneto-optical imaging of superconducting thin films, Supercond. Sci. Technol. 29, 035014 (2016).

[54] F. S. Wells, A. V. Pan, I. A. Golovchanskiy, S. A. Fedoseev, and A. Rozenfeld, Observation of transient overcritical currents in YBCO thin films using high-speed magneto-optical imaging and dynamic current mapping, Sci. Rep. 7, 40235 (2017).

[55] C. P. Bean, Magnetization of high-field superconductors, Rev. Mod. Phys. 36, 31 (1964).

[56] W. T. Norris, Calculation of hysteresis losses in hard superconductors carrying AC: Isolated conductors and edges of thin sheets, J. Phys. D: Appl. Phys. 3, 489 (1969). 
[57] D.-X. Chen and R. B. Goldfarb, Kim model for magnetization of type-II superconductors, J. Appl. Phys. 66, 2489 (1989).

[58] I. A. Golovchanskiy, A. V. Pan, O. V. Shcherbakova, and S. A. Fedoseev, Rectifying differences in transport, dynamic, and quasi-equilibrium measurements of critical current density, J. Appl. Phys. 114, 163910 (2013).

[59] M. Tinkham, Resistive Transition of High-Temperature Superconductors, Phys. Rev. Lett. 61, 1658 (1988).

[60] I. A. Golovchanskiy, A. V. Pan, J. George, F. S. Wells, S. A. Fedoseev, and A. Rozenfeld, Vibration effect on magnetization and critical current density of superconductors, Supercond. Sci. Technol. 29, 075002 (2016).

[61] K. Jeon, C. Ciccarelli, H. Kurebayashi, L. F. Cohen, X. Montiel, M. Eschrig, T. Wagner, S. Komori, A. Srivastava, J. W. A. Robinson, and M. G. Blamire, Effect of Meissner Screening and Trapped Magnetic Flux on Magnetization Dynamics in Thick $\mathrm{Nb} / \mathrm{Ni}_{80} \mathrm{Fe}_{20} / \mathrm{Nb}$ trilayers, Phys. Rev. Appl. 11, 014061 (2019).

[62] D. F. Gibbons and V. G. Chirba, Acoustical loss and Young's modulus of yttrium iron garnet, Phys. Rev. 110, 770 (1958).
[63] Kai Wang and Robert R. Reeber, The role of defects on thermophysical properties: Thermal expansion of $\mathrm{V}, \mathrm{Nb}$, Ta, Mo and W, Mater. Sci. Eng. R23, 101 (1998).

[64] S. Geller, G. P. Espinosa, and P. B. Crandall, Thermal expansion of yttrium and gadolinium iron, gallium and aluminum garnets, J. Appl. Cryst. 2, 86 (1969).

[65] Rui Sheng Liang and Feng Chao Liu, Measurement of thermal expansion coeffiecient of substrate GGG and its epitaxial layer YIG, Powder Diffr. 14, 2 (1999).

[66] H. J. Levinstein, E. M. Gyorgy, and R. C. LeCraw, Thermal expansion of YIG and YIG with $\mathrm{Mn}$ and Si additions, J. Appl. Phys. 37, 2197 (1966).

[67] A. M. Antyukhov, A. A. Sidorov, I. A. Ivanov, and A. V. Antonov, Thermal expansion coefficients of crystals of certain garnets over the range 6-310 K, Inorg. Mater. (Translated from Izv. Akad. Nauk SSSR, Neorg. Mater.) 23, 702 (1987).

[68] Tso Yee Fan, Daniel J. Ripin, Roshan L. Aggarwal, Juan R. Ochoa, Bien Chann, Michael Tilleman, and Joshua Spitzberg, Cryogenic $\mathrm{Yb}^{3+}$-doped solid-state lasers, IEEE J. Sel. Top. Quantum. Electron. 13, 448 (2007). 